\title{
Incidence of Major Adverse Cardiovascular and/or Limb Events among Patients Using Aspirin for Secondary Prevention
}

\author{
Nicholas J Leeper ${ }^{1}$, Windsor Ting ${ }^{2}$, Akshay Kharat ${ }^{3}$, Brian Murphy ${ }^{4}$, Jeffrey S Berger ${ }^{5}$, Alex Simpson ${ }^{6}$ and Ariel \\ Berger $^{4 *}$
}

${ }^{1}$ Stanford University Medical School, California, USA

${ }^{2}$ Mount Sinai Health System, New York, USA

${ }^{3}$ Real World Value \& Evidence, Cardiovascular, Janssen Scientific Affairs LLC, Titusville, NJ, US

${ }^{4}$ Real-World Evidence, Evidera, Waltham, MA, USA

${ }^{5}$ Center for the Prevention of Cardiovascular Disease, New York University School of Medicine, New York, USA

${ }^{6}$ Real-World Evidence, Evidera, Hammersmith, London, UK

*Corresponding author: Ariel Berger Evidera, Real-World Evidence, Evidera, Waltham, MA, USA

\begin{abstract}
Background: Low-dose aspirin (ASA) is often used for secondary prevention of major adverse cardiovascular events (MACE) and/or major adverse limb events (MALE) in chronic coronary artery disease (CAD) or peripheral artery disease (PAD). The COMPASS trial demonstrated that adding rivaroxaban to low-dose ASA significantly decreased relative risk of these events. However, its absolute impact will depend on real-world effectiveness of ASA monotherapy, as clinical trials differ from clinical practice.

Objectives: To examine real-world rates of MACE and MALE among patients receiving ASA as secondary prevention.

Methods: We used a large US claims database linked to electronic medical records to identify patients with chronic CAD/PAD who were using ASA for secondary prevention. We then assessed occurrence of MACE and MALE from date chronic CAD/PAD was established to earliest of death, disenrollment, or end of study.

Results: A total of 1,285 patients met selection criteria (mean age: 69.6 years; $60.7 \%$ male). Over a mean follow-up of 2.3 years, $16.0 \%$ experienced MACE or MALE (7.8 events per 100 person-years). Patients were more likely to experience events than the "ASAonly" COMPASS arm, including MACE (11.7\% vs. 5.6\% in patients with CAD; $14.0 \%$ vs. $6.9 \%$ in patients with PAD) and MALE (e.g., critical limb ischemia: $12.9 \%$ vs. $1.0 \%$ in PAD).

Conclusions: Real-world risk of MACE/MALE among patients using ASA as secondary prevention are substantially higher than that reported in the COMPASS trial. Further study is needed to determine whether emerging secondary prevention strategies have greater absolute impact on real-world clinical outcomes than those observed in clinical trials.

Keywords: Coronary Artery Disease; Peripheral Artery Disease; Secondary Prevention; Aspirin; Myocardial Infarction; Risk

Abbreviations: ASA: Aspirin; CAD: coronary artery disease; CCI: Charlson Comorbidity Index; CLI: critical limb ischemia: CPT-4: Current Procedural Terminology, Fourth Edition; CV: cardiovascular; DVT deep-vein thrombosis; ED: emergency department; EMR: electronic medical records; FDA: Food and Drug Administration; ICD-9-CM: International Classification of Diseases, Ninth Revision, Clinical Modification; MACE major adverse cardiovascular events; MALE: major adverse limb events; MI: myocardial infarction; OTC: over-the-counter; PAD: peripheral artery disease; PE: pulmonary embolism; PYs: person-years; SD: standard deviations
\end{abstract}




\section{Introduction}

Patients with coronary artery disease (CAD) and peripheral artery disease (PAD) are at elevated risk for build-up of atherosclerotic plaques. When ruptured, the sub-endothelial matrix is exposed to circulating blood, which in turn promotes the coagulation cascade and platelet aggregation, leading to arterial thrombi. Via this mechanism, patients with CAD or PAD are at high risk of experiencing major adverse cardiovascular events (MACE) (Bhatt et al., [1]) (e.g., stroke, myocardial infarction [MI], cardiovascular [CV] death) or major adverse limb events (MALE) (e.g., limb ischemia, amputation, arterial revascularization) (Gerhard-Herman etal., [2]). Low-dose (75-162 mg/d) aspirin (ASA) inhibits platelet aggregation and thus the risk of arterial thrombi (Antithrombotic Trialists Collaboration et al., [3]). When used as secondary prevention, it has been demonstrated to reduce risk of MACE by $20 \%$, including a $9 \%$ reduction in risk of CV death (each versus placebo) (Antithrombotic Trialists Collaboration et al., [3]). This risk reduction typically corresponds to an absolute reduction in annual incidence of fatal events of between $10-20$ per 1,000 , and a lesser, albeit still significant, reduction in vascular death and other serious events (Antithrombotic Trialists Collaboration[4]). The effectiveness of ASA in this context of secondary prevention is thus well established, and the American Heart Association currently assign it a level A, class I recommendation as secondary prevention for MACE and MALE among patients with known coronary heart disease or atherosclerotic vascular disease (Smith et al., [5]).

The COMPASS study was a large $(n=27,395)$, double-blind, double-dummy, randomized trial that compared rivaroxaban (a selective direct factor Xa inhibitor) in combination with low-dose ASA versus low-dose ASA alone for the prevention of secondary CV-related events among patients with chronic CAD and/or PAD (Eikelboom et al., [6]). Patients randomized to rivaroxaban (plus low-dose ASA) were less likely than those randomized to low-dose ASA alone to experience the composite outcome of MI, stroke and CV death (hazard ratio: 0.76; 95\% confidence interval 0.66-0.86; $\mathrm{p}<0.001$ ); the trial was stopped early as pre-specified criteria for superiority were attained earlier than anticipated. In part based on evidence from COMPASS, the United States Food and Drug Administration (FDA) has approved rivaroxaban (in combination with ASA) to reduce the risk of $\mathrm{CV}$ death, MI, and stroke among patients with chronic CAD or PAD (it is also indicated to reduce risk of stroke and systemic embolism in patients with nonvalvular atrial fibrillation; for treatment of deep-vein thrombosis [DVT] or pulmonary embolism [PE]; to reduce risk of recurrence of DVT and/or PE; as DVT prophylaxis which may lead to PE in patients undergoing knee or hip replacement; and, most recently, for prophylaxis of venous thromboembolism in acutely ill medical patients).

While rivaroxaban is a welcome addition to the armamentarium of secondary prevention among patients with existing CAD and PAD, the magnitude of its clinical benefit is likely at least in part dependent on the effectiveness of ASA as secondary prevention. For example, if most patients with prevalent disease currently receive secondary prevention with ASA-and if such therapy sufficiently reduces risk of MACE and/or MALE to an acceptably low level-clinician familiarity with ASA and its low acquisition cost (typically purchased as over-the-counter [OTC] medication in the US) will likely dissuade practitioners from prescribing additional therapies as prophylaxis. Conversely, should rates of MACE and/or MALE remain relatively high among patients treated with ASA, the importance of additional secondary preventionincluding add-on therapy with an agent such as rivaroxabanincreases. Consequently, to better understand the potential impact to clinical practice of this recent expansion of available secondary prevention options, we used a large US healthcare claims database linked to patients' electronic medical records (EMR) to examine the real-world incidence of MACE and/or MALE among patients with chronic CAD and PAD, who used ASA as secondary prevention. Our objective was to determine if the risk of MACE and MALE amongst real-world patients currently using an "ASA-only" approach justifies more aggressive secondary prevention measures.

\section{Materials and Methods}

\section{Data Source}

We used the Optum Integrated Database, which includes administrative healthcare data (i.e., claims, eligibility for medical and pharmacy benefits, demographic data) linked to EMR for approximately 5.5 million patients. Detailed information for each patient in the database includes: 1) primary and secondary diagnoses (in International Classification of Diseases, Ninth Revision, Clinical Modification [ICD-9-CM] and ICD-10-CM format); 2) procedures (in ICD-9-CM, ICD-10 CM, Healthcare Common Procedure Coding System (HCPCS), and Current Procedural Terminology, Fourth Edition [CPT-4] format); 3) test and lab results; 4) setting of care (e.g., inpatient, emergency department [ED]), and date[s] of service; 5) prescription dispenses from outpatient pharmacies; 6) use of OTC medications, noted in EMR; and 7) total reimbursed amounts for medical care and prescription pharmacotherapies. The database is fully de-identified, compliant with the Health Insurance Portability and Accountability Act (HIPAA) of 1996, and spanned the period January 1, 2009 to September 30, 2016 ("study period").

\section{Sample selection}

Patients were required to have $\geq 1$ outpatient visits with diagnoses of CAD or PAD between July 1, 2009 and September 29, 2016, and either: 1) evidence of hospital admissions or ED visits for CAD (or PAD) $>6$ months previously (the first outpatient visit $>6$ months from the last emergent care visit was designated the "index date") or 2) a second outpatient visit for CAD (or PAD) $\geq 30$ 
days subsequent to the initial outpatient visit, without evidence of emergent care for CAD (or PAD) between visits (the second outpatient visit was designated the "index date") (Figure 1). We focused attention on the period spanning July 1, 2009 to September 29,2016 so that all selected patients had $\geq 6$ months of pre-index information and a minimum of one day of follow-up. For patients with multiple index dates, we selected the earliest identified. Patients with $<6$ months enrolment prior to index date were dropped from the analyses, as were those: 1) with evidence of MACE or MALE within the 30-day period prior to index date; 2) who were aged $<18$ years at index date; 3 ) with evidence of atrial fibrillation during the 6-month pre-index period (these patients were dropped as anticoagulant therapy was already indicated for this condition at the time the work was conducted); 4) without evidence of use of ASA during pre-index period (evidence established through either $\geq 1$ relevant notations in patients' EMR or $\geq 1$ claims for such therapy [in the US, most ASA use is OTC, but physicians may write prescriptions for such therapy]); or 5) without claims and EMR data available during pre-index. Follow-up for each patient began on the index date and extended until the earliest of death, disenrollment from health plan, or end of study period. All available information from patients' claims and EMR were compiled for the pre-index period and all of follow-up.

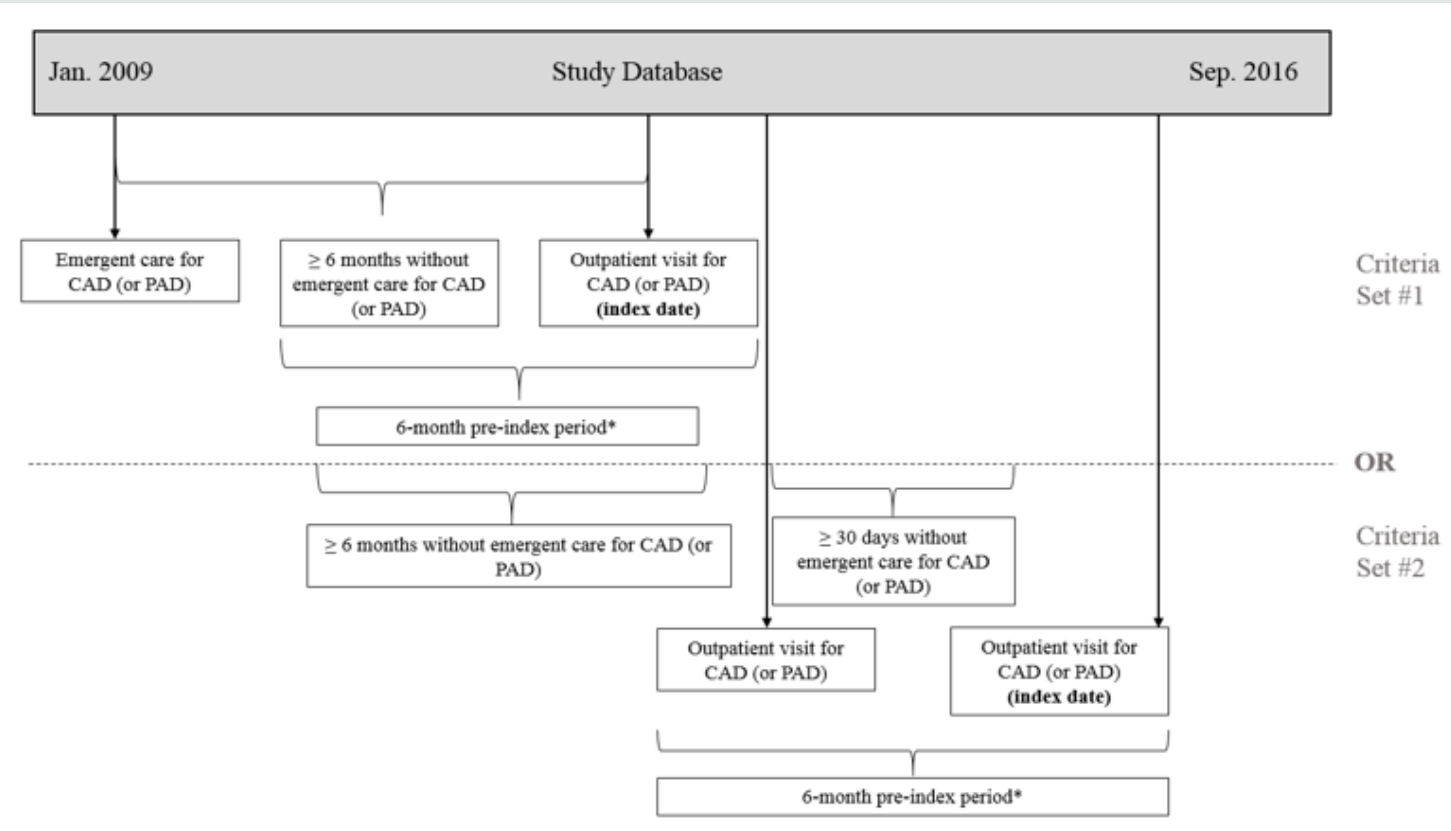

Figure 1: Illustration of Sample Selection.

* No evidence of emergent care for CAD (or PAD). Abbreviations: CAD = coronary artery disease, PAD = peripheral artery disease.

\section{Measures}

Demographic (e.g., age, gender, geographic region, race, insurance type), clinical characteristics (e.g., body mass index [BMI], selected comorbidities, Charlson Comorbidity Index [CCI] (Charlson, Szatrowski, Peterson, \& Gold, [7] as well as receipt of CVrelated prescription medications (e.g., anticoagulants, antiplatelets, antihypertensives) were based on all available information noted on the index date and through the pre-index period. Outcomes of interest during follow-up included MACE and MALE. The former was defined as the composite of MI, stroke (ischemic or hemorrhagic), or CV-related death (described in detail below); the latter, as critical limb ischemia (CLI), amputation (except for amputations related to accident/trauma), or PAD-related revascularization (definitions available upon request). Apart from revascularization or CV-related death, patients were required to have evidence of a hospital admission or ED visit during which a primary diagnosis specific to MACE/MALE (e.g., MI, CLI) was listed (we focused on the primary diagnosis as it represents the best reason to explain the hospitalization) ( $>90 \%$ of all identified events resulted in hospital admission). Incidence of revascularization was based on $\geq 1$ relevant procedure codes, irrespective of setting of care.

Mortality was assessed using specific information within the database (e.g., hospital discharge disposition, evidence of death recorded in the EMR, diagnosis of death [e.g., ICD-9-CM 348.82, ICD-10 G93.82]), augmented by methods described by Paramore and colleagues (Paramore, Thomas, Knopf, Cragin, \& Fraeman, [8]). Specifically, evidence during follow-up of a serious event (e.g., metastatic cancer, trauma, aortic dissection, pulmonary embolism) resulting in ambulance service or emergent care (i.e., hospital admission or ED visit) without subsequent claims for medical 
care or pharmacotherapy, and disenrollment the next month, were assumed to be fatal events. For all patients with evidence of death, those with CV-related diagnoses on the last date for which data were available (or during the admission where applicable) were assumed to have experienced CV-related death.

\section{Statistical analyses}

Pre-index measures were summarized using descriptive statistics (e.g., means, standard deviations [SDs] for continuous variables; numbers and percentages for categorical variables). The number and percentage of patients experiencing MACE and/ or MALE was estimated, as were the corresponding rates, which were expressed per 100 person-years (PYs) and were estimated by dividing the number who experienced the event by the total time at risk for the event (in years) and multiplying the result by 100. Incidence of both the composite events (i.e., MACE, MALE) and each corresponding component (e.g., MI, CLI) were estimated. Accordingly, patients could have different times at risk for each outcome. Attention was focused on the first instance of each outcome of interest during follow-up. In estimating risk of the composite outcome, the earliest relevant event was used (e.g., a PAD patient who experienced CLI and then amputation 8 months thereafter would contribute their time-at-risk for CLI to analyses of MALE). All analyses were conducted using SAS version 9.4 (SAS Institute Inc., Cary, North Carolina), and were run on the aggregate population as well as for the subgroups with CAD and PAD, respectively.

\section{Results}

A total of 1,285 patients had evidence of pre-index ASA use and met all inclusion and exclusion criteria, including 1,143 (88.9\%) with CAD and 278 (21.6\%) with PAD (138 patients had evidence of both CAD and PAD) (Table 1). Mean (SD) age of study subjects was 69.6 (10.6) years; $60.7 \%$ were men, $78.6 \%$ were white, and $66.2 \%$ had Medicare insurance (Table 2). Comorbidities were common, including hypertension (74.6\% had evidence of this condition), dyslipidemia (70.0\%), diabetes (32.3\%), malignancy (23.7\%) and congestive heart failure (15.2\%). In comparison to "ASA-only" COMPASS enrollees, patients in our study were nominally older (69.6 vs. 68.2 years in COMPASS), nominally more likely to be women (39.3\% vs. $21.8 \%)$, and nominally more likely to be white (78.6\% vs. 62.3\%); ASA-only COMPASS enrollees also had nominally greater levels of hypertension, CHF and prior stroke.

Table 1: Sample Selection.

\begin{tabular}{|c|c|c|c|}
\hline Selection Criteria & CAD Cohort & PAD Cohort & Aggregate Cohort \\
\hline$\geq 1$ outpatient encounters (excluding ED visits) for CAD/PAD & 280,850 & 112,600 & 330,978 \\
\hline $\begin{array}{c}\text { Continuous enrolment } 6+\text { months prior to CAD/PAD outpatient } \\
\text { encounter }\end{array}$ & 170,903 & 67,119 & 201,521 \\
\hline CAD/PAD outpatient encounter qualified as index date & 102,247 & 30,916 & 117,831 \\
\hline $\begin{array}{l}\text { Qualifying cohort group: No ED visits or admissions for CAD/PAD within } \\
6 \text { months of first relevant outpatient encounter for CAD/PAD }\end{array}$ & 88,378 & 29,825 & 105,568 \\
\hline $\begin{array}{l}\text { Qualifying cohort group: ED visits or admissions for CAD/PAD within } 6 \\
\text { months of first relevant outpatient encounter for CAD/PAD }\end{array}$ & 13,869 & 1,091 & 14,762 \\
\hline \multicolumn{4}{|l|}{ Total of above } \\
\hline $\begin{array}{l}\text { Without evidence of MACE or MALE events within } 30 \text { days prior to index } \\
\text { date and }\end{array}$ & 102,119 & 30,854 & 117,694 \\
\hline Aged $<18$ years on the index date and & 102,053 & 30,843 & 117,618 \\
\hline No evidence of atrial fibrillation during the 6-month pre-index period and & 85,754 & 25,695 & 99,730 \\
\hline Evidence of ASA use during the 6-month pre-index period & 1,143 & 278 & 1,285 \\
\hline Total number of patients qualifying for cohort & 1,143 & 278 & 1,285 \\
\hline
\end{tabular}

*All values are number of patients

Abbreviations: ASA: Aspirin, CAD: Coronary artery disease, MACE: Major adverse cardiovascular event, MALE: Major adverse limb event, PAD: Peripheral artery disease. 
Table 2: Demographic and clinical characteristics of patients with pre-index use of ASA, by cohort.

\begin{tabular}{|c|c|c|c|c|}
\hline & Any CAD & Any PAD & Aggregate & COMPASS ASA only arm \\
\hline & $(\mathrm{N}=1,143)$ & $(\mathrm{N}=278)$ & $(\mathrm{N}=1,285)$ & $(N=9,126)$ \\
\hline Mean (SD) age, years & $69.4(10.4)$ & $71.1(10.5)$ & $69.6(10.6)$ & $68.2(8.0)$ \\
\hline Male & $719(62.9)$ & $145(52.2)$ & $780(60.7)$ & $7,137(78.2)$ \\
\hline \multicolumn{5}{|c|}{ Race } \\
\hline White & $908(79.4)$ & $203(73.0)$ & $1,010(78.6)$ & $5,682(62.3)$ \\
\hline African American & $73(6.4)$ & $24(8.6)$ & $83(6.5)$ & $92(1.0)$ \\
\hline Asian & $23(2.0)$ & $5(1.8)$ & $26(2.0)$ & $1,397(15.3)$ \\
\hline All other & $139(12.2)$ & $46(16.5)$ & $166(12.9)$ & $1,955(21.4)$ \\
\hline \multicolumn{5}{|c|}{ Payer type } \\
\hline Commercial & $393(34.4)$ & $88(31.7)$ & $434(33.8)$ & N/A \\
\hline Medicare & $750(65.6)$ & $190(68.3)$ & $851(66.2)$ & $\mathrm{N} / \mathrm{A}$ \\
\hline All other & $0(0.0)$ & $0(0.0)$ & $0(0.0)$ & $\mathrm{N} / \mathrm{A}$ \\
\hline \multicolumn{5}{|c|}{ Geographic region } \\
\hline Northeast & $268(23.4)$ & $87(31.3)$ & $312(24.3)$ & N/A \\
\hline Midwest & $354(31.0)$ & $66(23.7)$ & $392(30.5)$ & N/A \\
\hline South & $416(36.4)$ & $85(30.6)$ & 459 (35.7) & N/A \\
\hline West & $85(7.4)$ & $31(11.2)$ & $97(7.5)$ & N/A \\
\hline \multicolumn{5}{|c|}{ Body mass index } \\
\hline$<18.5$ & $15(1.3)$ & $1(0.4)$ & $13(1.0)$ & NR \\
\hline $18.5-24.9$ & $155(13.6)$ & $58(20.9)$ & $189(14.7)$ & $\mathrm{NR}$ \\
\hline $25-29.9$ & $312(27.3)$ & $67(24.1)$ & $351(27.3)$ & $\mathrm{NR}$ \\
\hline$>30$ & $365(31.9)$ & $70(25.2)$ & $400(31.1)$ & $\mathrm{NR}$ \\
\hline Missing/Unknown & $296(25.9)$ & $82(29.5)$ & $332(25.8)$ & $\mathrm{NR}$ \\
\hline \multicolumn{5}{|c|}{ Smoking status } \\
\hline Current smoker & $217(19.0)$ & $81(29.1)$ & $267(20.8)$ & $1,972(21.6)$ \\
\hline Former smoker & $397(34.7)$ & $76(27.3)$ & $429(33.4)$ & NR \\
\hline Never smoked & $176(15.4)$ & $28(10.1)$ & $194(15.1)$ & $\mathrm{NR}$ \\
\hline Missing/Unknown & $353(30.9)$ & $93(33.5)$ & $395(30.7)$ & $\mathrm{NR}$ \\
\hline \multicolumn{5}{|c|}{ Comorbidities } \\
\hline Hypertension & $856(74.9)$ & $211(75.9)$ & $959(74.6)$ & $6,877(75.4)$ \\
\hline Dyslipidemia & $804(70.3)$ & $199(71.6)$ & $899(70.0)$ & NR \\
\hline Diabetes & $362(31.7)$ & $119(42.8)$ & $415(32.3)$ & $3,474(38.1)$ \\
\hline Malignancy & $277(24.2)$ & $62(22.3)$ & $304(23.7)$ & NR \\
\hline Chronic pulmonary disease & $221(19.3)$ & $63(22.7)$ & $247(19.2)$ & NR \\
\hline $\mathrm{CHF}$ & $186(16.3)$ & $43(15.5)$ & $195(15.2)$ & $1,979(21.7)$ \\
\hline Prior stroke & $38(3.3)$ & $12(4.3)$ & $46(3.6)$ & $335(3.7)$ \\
\hline CABG & $12(1.0)$ & $5(1.8)$ & $13(1.0)$ & NR \\
\hline $\mathrm{PCI}$ & $19(1.7)$ & $3(1.1)$ & $21(1.6)$ & $\mathrm{NR}$ \\
\hline \multicolumn{5}{|c|}{$\geq 1 \mathrm{CV}$-related prescription medications $\uparrow$} \\
\hline Anticoagulants & $36(3.1)$ & $3(1.1)$ & $38(3.0)$ & NR \\
\hline Antiplatelets & $11(1.0)$ & $14(5.0)$ & $21(1.6)$ & NR \\
\hline Antihypertensives & $707(61.9)$ & $177(63.7)$ & $786(61.2)$ & NR \\
\hline Cholesterol-modifying agents & $604(52.8)$ & $150(54.0)$ & $682(53.1)$ & NR \\
\hline Nitroglycerin & $113(9.9)$ & $17(6.1)$ & $111(8.6)$ & $\mathrm{NR}$ \\
\hline Pentoxifylline & $3(0.3)$ & $3(1.1)$ & $5(0.4)$ & $\mathrm{NR}$ \\
\hline Any CV-related prescriptions & $777(68.0)$ & $203(73.0)$ & $875(68.1)$ & NR \\
\hline
\end{tabular}

*Unless otherwise noted, all variables are $\mathrm{n}(\%)$.

†Defined as $\geq 1$ prescription dispenses for, or administrations of, relevant medication during pre-index period

Abbreviations: ASA: Aspirin; BMI: Body mass index; CABG: Coronary artery bypass grafting; CAD: Coronary artery disease; CHF: Congestive heart failure; CV: Cardiovascular; PAD: Peripheral artery disease; PCI: Percutaneous coronary intervention; SD: Standard deviation. 
Over a mean follow-up of 2.3 years, $16.0 \%$ of study subjects experienced $\geq 1$ events of interest, (predominantly MACE), resulting in a rate of 7.8 events per 100 PYs. A total of 5.2\% (2.4 per $100 \mathrm{PYs}$ ), 4.4\% (2.0 per 100 PYs), 4.9\% (2.2 per 100 PYs), and 11.8\% (5.5 per 100 PYs) of study subjects experienced MI, stroke, CV death, or any of these events (i.e., MACE), respectively (Table 3). This contrasts the much lower event rates observed in COMPASS, where 2.2\% (1.2 per $100 \mathrm{PYs}$ ), $1.6 \%$ (0.8 per $100 \mathrm{PYs}$ ), $2.2 \%$ (1.2 per $100 \mathrm{PYs}$ ), and $5.4 \%$ (2.9 per PYs) ASA-only enrollees experienced MI, stroke, CV death, and the composite of these events over a mean follow-up of 1.9 years.

Table 3: Incidence of MACE and MALE events among patients with chronic CAD and/or PAD and pre-index use of ASA, by condition.

\begin{tabular}{|c|c|c|c|c|c|c|c|c|c|c|c|c|}
\hline & \multicolumn{4}{|c|}{ CAD } & \multicolumn{4}{|c|}{ PAD } & \multicolumn{4}{|c|}{ Aggregate } \\
\hline & \multirow{2}{*}{\multicolumn{2}{|c|}{$\begin{array}{c}\text { Current Study } \\
(\mathrm{N}=1,143)\end{array}$}} & \multirow{2}{*}{\multicolumn{2}{|c|}{$\begin{array}{c}\text { COMPASS ASA Only } \\
(\mathrm{N}=\mathbf{8 , 2 6 1})\end{array}$}} & \multirow{2}{*}{\multicolumn{2}{|c|}{$\begin{array}{c}\text { Current Study } \\
(\mathrm{N}=\mathbf{2 7 8})\end{array}$}} & \multirow{2}{*}{\multicolumn{2}{|c|}{$\begin{array}{c}\text { COMPASS ASA Only } \\
(\mathrm{N}=2,504)\end{array}$}} & \multirow{2}{*}{\multicolumn{2}{|c|}{$\begin{array}{c}\text { Current Study } \\
(\mathrm{N}=1,285)\end{array}$}} & \multirow{2}{*}{\multicolumn{2}{|c|}{$\begin{array}{c}\text { COMPASS ASA Only } \\
(\mathrm{N}=9,126)\end{array}$}} \\
\hline & & & & & & & & & & & & \\
\hline & N (\%) & Rate* & N (\%) & Rate* & N (\%) & Rate* & N (\%) & Rate* & N (\%) & Rate* & N (\%) & Rate* \\
\hline \multicolumn{13}{|c|}{ MACE } \\
\hline MI & $58(5.1)$ & 2.3 & $195(2.4)$ & NR & $13(4.7)$ & 2.4 & $67(2.7)$ & 1.5 & $67(5.2)$ & 2.4 & $205(2.2)$ & 1.2 \\
\hline Stroke & $50(4.4)$ & 2.0 & $130(1.6)$ & NR & $14(5.0)$ & 2.5 & 47 (1.9) & 1.0 & $56(4.4)$ & 2.0 & $142(1.6)$ & 0.8 \\
\hline CV death & $57(5.0)$ & 2.2 & $184(2.2)$ & NR & $18(6.5)$ & 3.2 & $78(3.1)$ & 1.7 & $63(4.9)$ & 2.2 & $203(2.2)$ & 1.2 \\
\hline Any of above & $\begin{array}{c}134 \\
(11.7)\end{array}$ & 5.4 & $460(5.6)$ & NR & $39(14.0)$ & 7.2 & $173(6.9)$ & 3.9 & $\begin{array}{c}151 \\
(11.8)\end{array}$ & 5.5 & $\begin{array}{c}496 \\
(5.4) \dagger\end{array}$ & 2.9 \\
\hline \multicolumn{13}{|c|}{ MALE } \\
\hline CLI & $21(1.8)$ & 0.8 & NR & NR & 36 (12.9) & 7.2 & $24(1.0)$ & 0.5 & $44(3.4)$ & 1.6 & NR & NR \\
\hline $\begin{array}{l}\text { Vascular } \\
\text { amputation }\end{array}$ & $3(0.3)$ & 0.1 & NR & NR & $7(2.5)$ & 1.3 & $28(1.1)$ & 0.6 & $7(0.5)$ & 0.2 & NR & NR \\
\hline Revascularization & $26(2.3)$ & 1.0 & NR & NR & $20(7.2)$ & 3.8 & NR & NR & $40(3.1)$ & 1.4 & NR & NR \\
\hline Any of above & $41(3.6)$ & 1.6 & NR & NR & 48 (17.3) & 10.2 & $60(2.4)$ & 1.3 & $72(5.6)$ & 2.6 & NR & NR \\
\hline $\begin{array}{c}\text { Any MACE or MALE } \\
\text { event }\end{array}$ & $\begin{array}{c}163 \\
(14.3)\end{array}$ & 6.8 & $\begin{array}{c}470 \\
(5.7) \dagger\end{array}$ & NR & 77 (27.7) & 16.8 & $225(9.0)$ & 5.1 & $\begin{array}{c}205 \\
(16.0)\end{array}$ & 7.8 & $\begin{array}{c}516 \\
(5.7) \neq\end{array}$ & NR \\
\hline
\end{tabular}

*Expressed in terms of events per 100 PYs at risk

†Represents the COMPASS primary outcome of MI, ischemic stroke, cardiovascular death, or acute limb ischemia.

$\ddagger$ Represents the COMPASS secondary outcome of MI, ischemic stroke, CV death or CLI; vascular amputation and revascularization were not available for this subgroup in COMPASS. ASA: Aspirin; CAD: Coronary artery disease; CLI: Critical limb ischemia; CV: Cardiovascular; MACE: Major adverse cardiac event; MALE: Major adverse limb event; MI: myocardial infarction; NR: Not reported; PAD: Peripheral artery disease; PYs: Person-years

Over comparable periods of follow-up (i.e., 2.3 years in our study vs. 1.9 years for COMPASS), incidence of MACE among subjects with CAD in our study was substantially greater than that reported in COMPASS, including MI (5.1\% of CAD patients in our study vs. $2.4 \%$ of those with CAD enrolled in COMPASS), stroke (4.4\% vs. $1.6 \%)$, CV death (5.0\% vs. $2.2 \%)$ and the composite of these events $(11.7 \%$ vs. 5.6\%). Similarly, those with PAD in our study also were more likely to experience events than comparable enrollees in COMPASS. For MACE-related events, this included MI (4.7\% vs. $2.7 \%$ of PAD enrollees randomized to ASA-only in COMPASS), stroke (5.0\% vs. $1.9 \%$ ), CV death (6.5\% vs. $3.1 \%$ ), and the composite of these events (14.0\% vs. 6.9\%); for MALE-related events, this included CLI (12.9\% vs. $1.0 \%)$ and vascular amputation (2.5\% vs. $1.1 \%)$. Nearly $28 \%$ of patients with PAD in our study experienced MACE or MALE, as compared with $9.0 \%$ of enrollees with PAD in the ASA-only arm in COMPASS (Anand et al., 2018).

\section{Discussion}

In our real-world cohort of patients with prevalent and chronic CAD or PAD who were using ASA as secondary prophylaxis, approximately one-in-six patients experienced MACE and/or MALE annually, equating to a rate of 7.8 per 100 PYs. Incidence of MACE and MALE events were substantially greater than that observed among subjects randomized to the "ASA-only" arm in the COMPASS trial. Our findings are consistent with, albeit of a different magnitude than, those reported by Darmon and colleagues, who found rates of the primary outcome examined in COMPASS (i.e., a composite of MI, stroke, and CV death) to be approximately $50 \%$ higher among participants in the Reduction of Atherothrombosis for Continued Health (REACH) registry than in those randomized to ASA-only in COMPASS (Darmon et al., 2018) . The risk of MACE and/or MALE observed in our study also was about twice that reported in a large meta-analysis of 16 secondary prevention trials 
that did not include COMPASS (which had not yet begun at the time of publication). The Antithrombotic Trialists Collaboration, which included results from 17,000 high-risk CV patients who collectively contributed 43,000 years of person-time, found that in clinical trials, $6.7 \%$ of patients treated with ASA experience MACE annually (rate $=3.5$ per 100 PYs) (Antithrombotic Trialists Collaboration et al., [3]). While we found substantially higher rates of MACE and MALE associated with ASA use than these studies, we note that the latter are from randomized trials, and therefore based on patients who likely differ in important ways from those typically seen in clinical practice. Indeed, one review of selection criteria used in clinical trials found that $>50 \%$ of patients seen in clinical practice would not meet selection criteria for inclusion in major cardiological RCTs for various reasons, including older age, presence of clinical impairment and/or comorbidities (less common in trial enrollees), and limited likelihood of treatment with guideline-recommended therapies (Kennedy-Martin, Curtis, Faries, Robinson, \& Johnston, [9]). Patients enrolled in clinical trials also differ from those in clinical practice in that the former typically enjoy protocol-mandated care, including relatively frequent visits and check-ins, which may positively impact their overall health in a number of unmeasurable and/or difficult to quantify ways relative to the latter. Importantly, a recent study that compared eligibility criteria across trials of 12 therapies intended to prevent major cardiovascular events among patients with known ischemic heart disease $(n=6,292)$ or prior MI $(n=2,277)$ found that selection criteria for the COMPASS trial were least restrictive (e.g., patients enrolled in COMPASS were most like those in clinical practice relative to the other cardiovascular-related trials included) (Mortensen, Blaha, \& Nordestgaard, [10]).

These differences also raise questions into whether ASA only is sufficient as secondary prevention. Given that in COMPASS rivaroxaban plus low-dose ASA reduced the risk of MACE (the primary outcome of COMPASS) by $24 \%$ (vs. low-dose ASA alone), a comparable outcome (assuming comparable real-world performance of this combination regimen to that reported in COMPASS) would be a reduction of MACE from 11.8\% $(n=151)$ of patients in clinical practice to $9.0 \%(\mathrm{~N}=115)$. However, we note that such a risk reduction would also be in tandem with an increase in risk of major bleeding (fatal bleeding, bleeding into a critical organ, bleeding into a surgical site requiring reoperation and bleeding that led to hospitalization) (subjects randomized to receive rivaroxaban plus low-dose ASA had a 1.7-fold increased risk of major bleeding vs. those randomized to ASA-only) (Eikelboom et al., [6]). Despite this increased risk, COMPASS found a positive net clinical benefit of combination therapy with rivaroxaban plus low-dose ASA (i.e., a lower risk of the composite outcome of CV death, stroke, MI, or fatal or symptomatic bleeding with combination therapy vs. ASA alone [hazard ratio $0.80 ; \mathrm{p}<0.001]$ ). Such risk/benefit evaluations should be evaluated on a per-patient basis to ensure optimal levels of care, which can vary patient-to-patient based on a number of criteria, are rendered.

Other retrospective observational studies investigating the performance of ASA as secondary prevention among patients with established CAD or PAD appear to be lacking. Information from the Reduction of Atherothrombosis for Continued Health (REACH) registry has been used to estimate rates of MACE or MALE among persons with existing CAD or PAD. In one study based on data from the REACH registry that focused on 65,531 "COMPASS-like" patients who were using ASA as secondary prevention, the investigators reported 4.2 MACE per 100 PYs (Darmon et al., [11]). While this reported rate is nominally lower than the 5.5 per 100 PYs observed among patients receiving ASA in our study, it is consistent with our findings in that it is numerically higher than the 2.9 per 100 PYs reported in COMPASS (Darmon et al., [11]). While conjectural, it is possible that application of COMPASS selection criteria may have resulted in exclusion of some patients at relatively high risk for MACE or MALE (i.e., the rate of these events among all REACH participants may be greater than that estimated among the "COMPASS-like" subgroup); the authors in fact note that application of the COMPASS selection criteria resulted in the exclusion of almost one-half of registry enrollees (47.1\%). Accordingly, we believe that our findings are consistent with those reported by the REACH investigators and provide additional evidence suggesting that among patients with chronic CAD and/or PAD seen in clinical practice, ASA monotherapy may be insufficient protection against MACE and MALE. Selection criteria for randomized clinical trials are often fairly stringent, with potentially concerning implications concerning their generalizability to real-world patients. Although registry studies such as REACH and the prospeCtive observational LongitudinAl RegIstry oF patients with stable coronary arterY disease (CLARIFY) are more representative of real-world settings, their selection criteria may still not approximate the range of patients seen in clinical practice (e.g., REACH was limited to patients aged $\geq 45$ years with either $\geq 3$ atherothrombotic risk factors or documented cerebrovascular disease, CAD) (Ohman et al., [12]).

Our analyses of a large US healthcare claims database linked to patients' EMR may provide a more comprehensive picture of patients seen in real-world practice. Further real-world research is warranted to better understand the clinical benefit associated with use of rivaroxaban plus low-dose ASA among patients with chronic CAD and/or PAD seen in clinical practice. Secondary prevention of MACE and MALE is an important objective for clinicians and their patients, and ASA has long been recommended as a means by which this goal can be attained (Smith et al., 2011). Despite its low cost and longevity, the latter of which has led to familiarity by physicians and their patients, use of low-dose ASA has been reported to range from $30 \%$ to $86.9 \%$ of patients with existing heart disease 
(Danchin et al.,; Fang et al., ; Stuntz \& Bernstein, [13-15]). In the aforementioned REACH study, 79\% of "COMPASS-eligible" patients had evidence of ASA use (Darmon et al., [13]); however, self-reported ASA use has declined between calendar-years 2012 and 2015from $22.8 \%$ to $20.4 \%$ (Stuntz \& Bernstein, [15]). Another examination, this one of patients with PAD that was based on the National Health and Nutrition Examination Survey (NHANES) found that $64.2 \%$ reported that they did not use ASA for secondary prevention; overall, between $61 \%$ and $75 \%$ reported not using any pharmacotherapies for secondary prevention (Pande, Perlstein, Beckman, \& Creager, [16]). This is consistent with well-known risks of non-adherence-the American Hospital Association has estimated that $12 \%$ of patients that are prescribed therapies do not fill their prescriptions (American Heart Association). While unknowable from our database, these and related challenges for secondary prevention may help explain to at least some degree the relatively high rates of MACE and MALE we observed in a real-world population of chronic with CAD and PAD. Regardless, these challenges-and our findings-serve as a stark reminder to clinicians of the importance of effective secondary prevention.

Like any observational study, ours has several limitations that should be noted. First is the issue of missing data among our initial CAD and PAD patient sample. While we initially identified 99,730 patients with CAD or PAD who met all other selection criteria, only 1,285 (1.3\%) had evidence of receipt of ASA. While Other observational studies have confirmed that ASA use may be under recorded (Berger \& Ladapo, [17]); accordingly, it is likely that ASA use was more widespread among patients in our sample than available data would indicate. Many patients who use ASA for secondary prevention likely use over-the-counter (OTC) (as opposed to prescription) preparations, and our study focused on those with prevalent CAD/PAD, which means that the relevant information on OTC products may not have been recorded during the study period. However, the patients who used ASA in our sample were likely to be "true positives", as they all had evidence of use of such therapy-predominantly from information available in their EMR. Given the goals of our study, we thought it important to maximize specificity (i.e., risk not including all "true positives") in order to limit the possibility of including patients who did not use ASA for secondary prevention (i.e., "false positives"). While reasons for omission of this information from the EMR are unknowable, we note that rates of MACE and MALE were comparable between those with and without evidence of pre-index ASA use (data not shown). Even if all patients in our sample did in fact use ASA for secondary prevention, the relatively high rates of MACE and MALE across our sample (as compared with either patients randomized to ASA in COMPASS and other trials or real-world experience as described by REACH) indicate that the effectiveness of such therapy in preventing CV events in clinical practice is suboptimal. Second, both claims and EMRs are subject to errors of omission and/or commission, with unknowable impact to our study. Third, and as noted above, our decision to include almost all patients with evidence of chronic CAD and/or PAD resulted in a sample who have lived with $\mathrm{CAD}$ or PAD for varying lengths of time, with resulting impacts on disease severity/risks of CV events (although we note that all patients were required to have not experienced either ED visits or hospitalizations resulting in diagnoses of CAD or PAD for at least 6 months prior to index date). Accordingly, while our sample likely reflects the mix of patients in typical clinical practice, resulting estimates of MACE and MALE reflect a somewhat "average" rate across varying patient-types. Fourth, we focused our attention on the first occurrence of each event during the follow-up period, and thus are likely underestimating "true" rates of MACE and MALE (i.e., our findings are likely conservative in terms of the current risk of MACE and/or MALE among patients using ASA for secondary prevention). Finally, while large and geographically diverse, ours was a convenience-not a random-sample. Consequently, the generalizability of our findings to all patients with chronic CAD and/or PAD is unknown[18,19].

\section{Conclusion}

In conclusion, rates of MACE and MALE among patients in clinical practice with chronic CAD and/or PAD who use ASA for secondary prevention are substantially greater than those reported in the COMPASS trial. Our findings reinforce that low-dose ASA monotherapy may be a suboptimal secondary prevention strategy in clinical practice. Further study is needed to understand the degree to which alternative management strategies-including but not limited to the addition of rivaroxaban to low-dose ASAmay reduce the incidence and burden of MACE and MALE among patients with chronic CAD and/or PAD in real-world settings.

\section{References}

1. Bhatt DL, Eagle KA, Ohman EM, Hirsch AT, Goto S, Mahoney EM (2010) Comparative determinants of 4-year cardiovascular event rates in stable outpatients at risk of or with atherothrombosis. JAMA 304(12): 13501357.

2. Gerhard-Herman MD, Gornik HL, Barrett C, Barshes NR, Corriere MA, et al. (2017) 2016 AHA/ACC Guideline on the Management of Patients With Lower Extremity Peripheral Artery Disease: A Report of the American College of Cardiology/American Heart Association Task Force on Clinical Practice Guidelines. Circulation 135(12): e726-e779.

3. Antithrombotic Trialists Collaboration, Baigent C, Blackwell L, Collins R, Emberson J, et al. (2009) Aspirin in the primary and secondary prevention of vascular disease: collaborative meta-analysis of individual participant data from randomised trials. Lancet 373(9678): 1849-1860.

4. Antithrombotic Trialists Collaboration (2002) Collaborative metaanalysis of randomised trials of antiplatelet therapy for prevention of death, myocardial infarction, and stroke in high risk patients. BMJ, 324(7329): 71-86.

5. Smith SC Jr, Benjamin EJ, Bonow RO, Braun LT, Creager (2011) AHA/ ACCF Secondary Prevention and Risk Reduction Therapy for Patients with Coronary and other Atherosclerotic Vascular Disease: 2011 update: a guideline from the American Heart Association and American College of Cardiology Foundation. Circulation, 124(22): 2458-2473. 
6. Eikelboom JW, Connolly SJ, Bosch J, Dagenais GR, Hart RG, et al. (2017) Rivaroxaban with or without Aspirin in Stable Cardiovascular Disease. N Engl J Med 377(14): 1319-1330.

7. Charlson M, Szatrowski TP, Peterson J, Gold J (1994) Validation of a combined comorbidity index. J Clin Epidemiol 47(11): 1245-1251.

8. Paramore LC, Thomas SK, Knopf KB, Cragin LS, Fraeman KH (2006) Estimating costs of care for patients with newly diagnosed metastatic colorectal cancer. Clin Colorectal Cancer 6(1): 52-58.

9. Kennedy-Martin T, Curtis S, Faries D, Robinson S, Johnston J (2015) A literature review on the representativeness of randomized controlled trial samples and implications for the external validity of trial results. Trials 16: 495.

10. Mortensen, MB, Blaha MJ, Nordestgaard BG (2020) Eligibility and Preventive Potential for New Evidence-Based Cardiovascular Drugs in Secondary Prevention. JAMA Cardiol 5(2): 209-215.

11. Darmon A, Bhatt DL, Elbez Y, Aboyans V, Anand S, et al. (2018) External applicability of the COMPASS trial: an analysis of the reduction of atherothrombosis for continued health (REACH) registry. Eur Heart J 39(9): 750-757a.

12. Ohman EM, Bhatt DL, Steg PG, Goto S, Hirsch AT, et al. (2006) The REduction of Atherothrombosis for Continued Health (REACH) Registry: An international, prospective, observational investigation in subjects at risk for atherothrombotic events-study design. American Heart Journal 151(4): 786.e781-786.e710.
13. Danchin N, Ferrieres J, Guenoun M, Cattan S, Rushton-Smith SK, et al. (2014) Management of outpatients in France with stable coronary artery disease. Findings from the prospeCtive observational Longitud in Al RegIstry of patients with stable coronary arterY disease (CLARIFY) registry. Arch Cardiovasc Dis, 107(8-9): 452-461.

14. Fang J, George MG, Gindi RM, Hong Y, Yang Q, et al. (2015) Use of lowdose aspirin as secondary prevention of atherosclerotic cardiovascular disease in US adults (from the National Health Interview Survey, 2012). Am J Cardiol 115(7): 895-900.

15. Stuntz M, Bernstein B (2017) Recent trends in the prevalence of lowdose aspirin use for primary and secondary prevention of cardiovascular disease in the United States, 2012-2015. Prev Med Rep 5: 183-186.

16. Pande RL, Perlstein TS, Beckman JA, Creager MA (2011) Secondary prevention and mortality in peripheral artery disease: National Health and Nutrition Examination Study, 1999 to 2004. Circulation 124(1): 1723.

17. Berger JS, Ladapo JA (2017) Underuse of Prevention and Lifestyle Counseling in Patients With Peripheral Artery Disease. J Am Coll Cardiol 69(18): 2293-2300.

18. American Heart Association. Statistics You Need To Know, Statistics on Medication. 107.

19. Anand SS, Bosch J, Eikelboom JW, Connolly SJ, Diaz R, et al. (2018). Rivaroxaban with or without aspirin in patients with stable peripheral or carotid artery disease: an international, randomised, double-blind, placebo-controlled trial. Lancet 391(10117): 219-229.

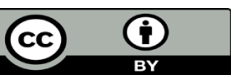

This work is licensed under Creative Commons Attribution 4.0 License

To Submit Your Article Click Here:

Submit Article

DOI: 10.32474/ACR.2020.03.000158

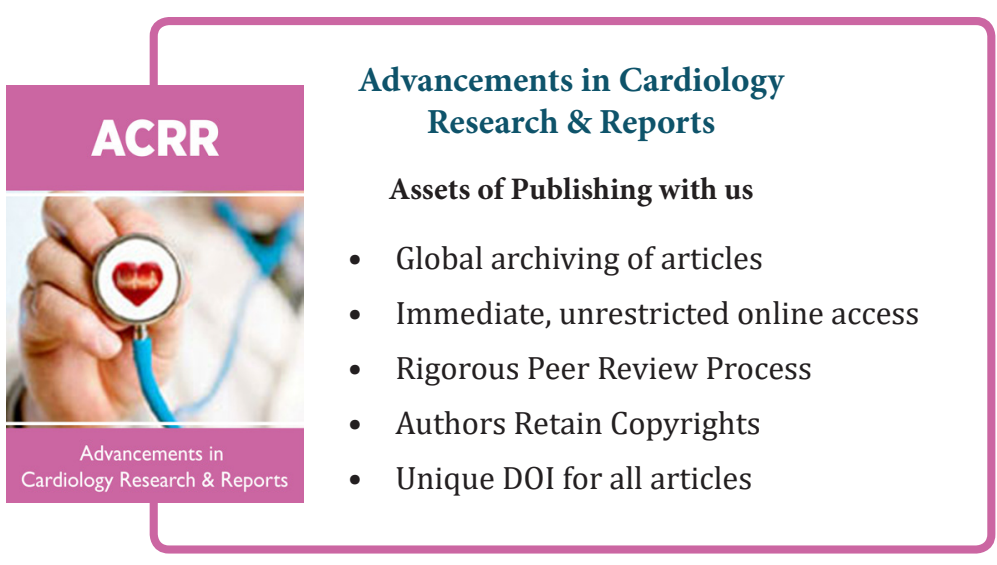

